\title{
Efficacy of Propranolol, Bisoprolol, and Pyridostigmine for Postural Tachycardia Syndrome: a Randomized Clinical Trial
}

\author{
Jangsup Moon ${ }^{1}$ - Do-Yong Kim ${ }^{1} \cdot$ Woo-Jin Lee ${ }^{1} \cdot$ Han Sang Lee ${ }^{1} \cdot J^{\prime}$ ung-Ah Lim ${ }^{2} \cdot$ Tae-Joon Kim ${ }^{1}$ - Jin-Sun Jun ${ }^{1}$. \\ Byeongsu Park ${ }^{3}$. Jung-Ick Byun ${ }^{4}$. Jun-Sang Sunwoo ${ }^{5}$. Soon-Tae Lee ${ }^{1} \cdot$ Keun-Hwa Jung ${ }^{1} \cdot$ Kyung-II Park ${ }^{6}$. \\ Ki-Young Jung ${ }^{1} \cdot$ Manho Kim ${ }^{1} \cdot$ Sang Kun Lee ${ }^{1} \cdot$ Kon Chu $^{1}$ (D)
}

Published online: 2 March 2018

(C) The American Society for Experimental NeuroTherapeutics, Inc. 2018

\begin{abstract}
Postural tachycardia syndrome (POTS) is a form of dysautonomia which presents with complex symptoms including orthostatic intolerance. Several medications are prescribed for POTS; however, the efficacy of sustained medical treatment has not been wellinvestigated. Here, we conducted a $2 \times 2$ factorial design, randomized, clinical trial of a 3-month medical treatment regimen in POTS patients. Patients were randomly allocated to 4 treatment groups (Group 1: propranolol; Group 2: bisoprolol; Group 3: propranolol + pyridostigmine; Group 4: bisoprolol + pyridostigmine). The orthostatic intolerance questionnaire (OIQ), Beck depression inventory-II (BDI-II), and short-form health survey (SF-36) were conducted at baseline, 1 and 3 months after treatment. Seventy-seven patients who completed the 3-month follow-up were analyzed. In total, every clinical score improved significantly after medical treatment. The OIQ score was significantly lower than that at baseline $(18.5 \pm 6.7)$ after 1 month $(12.5 \pm 4.5, P<0.01)$, which decreased further after 3 months $(7.8 \pm 5.7, P<0.01)$. The OIQ score improvements were consistent across every treatment group. In the subgroup analysis of 59 patients who did not receive antidepressants, the BDI-II score significantly decreased after treatment, regardless of the regimen. Physical components of the SF-36 improved after 3 months in every group, while mental components improved only in Group 3. The amount of changes in each score was similar among groups throughout the comparisons. Sustained medical treatment is beneficial to POTS patients, not only for orthostatic intolerance symptoms but also for depression and diminished quality of life, even without prescriptions for antidepressants. The efficacy of each regimen in POTS patients was comparable. Trial registration: NCT02171988
\end{abstract}

Electronic supplementary material The online version of this article (https://doi.org/10.1007/s13311-018-0612-9) contains supplementary material, which is available to authorized users.

Sang Kun Lee

sangkun2923@gmail.com

Kon Chu

stemcell.snu@gmail.com

1 Department of Neurology, Comprehensive Epilepsy Center, Laboratory for Neurotherapeutics, Biomedical Research Institute, Seoul National University Hospital, 101 Daehak-ro, Jongno-gu, Seoul 110-744, South Korea

2 Department of Neurology, Kangnam Sacred Heart Hospital, Hallym University College of Medicine, Seoul, South Korea

3 Department of Neurology, Ulsan University Hospital, Ulsan, South Korea

4 Department of Neurology, Kyung Hee University Hospital at Gangdong, Seoul, South Korea

5 Department of Neurology, Soonchunhyang University School of Medicine, Seoul, South Korea

6 Department of Neurology, Seoul National University Hospital Healthcare System Gangnam Center, Seoul, South Korea
Key Words Postural tachycardia syndrome $\cdot$ Randomized trial $\cdot$ Propranolol $\cdot$ Bisoprolol $\cdot$ Pyridostigmine

\section{Introduction}

Postural tachycardia syndrome (POTS), which is characterized by an excessive heart rate (HR) increase after standing, is a common cause of orthostatic intolerance (OI) [1-3]. POTS is more prevalent in young people and predominantly affects women (4-5:1) [4]. It is one of the most common disorders seen in autonomic dysfunction clinics and the prevalence is estimated to be at least $170 / 100,000$, which may be higher due to a lack of clinical awareness of the syndrome $[4,5]$. The clinical significance of POTS is increasingly appreciated due to the recent reports regarding the comorbidities of POTS, such as depression [1], sleep disorders [6], chronic fatigue syndrome [7], and diminished quality of life (QOL) [6, 8]. While the long-term prognosis of POTS has not been investigated well, some patients suffer from refractory POTS [9-11]. 
Both non-pharmacological and pharmacological treatments are being applied to POTS patients [12]. Water/ sodium ingestion and exercise are the mainstays of nonpharmacological treatments; however, POTS patients often report debilitated feelings after exercise, which limits their compliance with exercise regimens [12]. In terms of pharmacological treatments, $\beta$-blockers [13-16], pyridostigmine [12, 17], midodorine [18-21], and other agents [15, 22, 23] are used. A number of $\beta$-blockers including propranolol [13, 14], bisoprolol [15], and metoprolol [16] have been reported to be helpful in POTS patients; however, the superiority of efficacy among these agents has not been investigated. Pyridostigmine, an acetylcholinesterase inhibitor widely prescribed to POTS patients, is considered to increase parasympathetic nervous system activity and therefore decreased HR in POTS $[17,24]$. To date, the efficacy of combination treatment of $\beta$-blockers and pyridostigmine in POTS patients has not been investigated. Also, none of the abovementioned medications have been approved by the Food and Drug Administration for the treatment of POTS; thus, all agents are used off-label [12]. Furthermore, all trials have been of a short duration, and long-term, randomized, clinical trials in this area are lacking. Whether depression and diminished QOL would improve after treatment of POTS is also an important question that remains to be answered since these accompanying conditions significantly deteriorate the daily life of these patients.

Here, we performed a randomized, clinical trial of 3-month duration of medical treatments with 4 different regimens in POTS patients. We attempted to investigate the efficacy of sustained medical treatments, the superiority between two different $\beta$-blockers, and the benefit of additional pyridostigmine treatment. We also analyzed if the medical treatments for POTS were effective in improving depression and the diminished QOL.

\section{Methods}

\section{Subjects}

Patients who visited the Neurology department of Seoul National University Hospital between April 2014 and August 2015 and who satisfied the HR criteria for POTS were screened for eligibility for the current study. Orthostatic vital sign (OVS) tests were performed as previously described [2, 8]. The patients stayed in a supine position for $\geq 10 \mathrm{~min}$ before the test. After the baseline blood pressure (BP) and HR were measured, patients stood upright without support and remained still beside the bed. The BP and HR were checked immediately and again at $1,3,5$, and 10 min after standing. The OVS test was performed twice in every patient, mostly once in the afternoon and again in the early morning, and the maximal HR increment in each patient was calculated from the two OVS tests.

Patients were included in the study when they met the following criteria: (1) HR increment $\geq 30 \mathrm{bpm}$ (or $\geq 40 \mathrm{bpm}$ in patients aged between 12 and 19) within 10 min after standing; (2) presence of considerable orthostatic intolerance symptoms, defined by an orthostatic intolerance questionnaire (OIQ) [25] score $\geq 10$; and (3) no overt cause of tachycardia, such as acute blood loss, prolonged bed rest, hyperthyroidism, or tachycardia-promoting medications. This study was approved by the Institutional Review Board of Seoul National Hospital (IRB No. H-1401-091-440) and was registered at ClinicalTrials.gov (NCT02171988). Informed consent was obtained from every participant.

\section{Study Design}

Patients were randomly allocated to 4 different treatment groups (Group 1: propranolol; Group 2: bisoprolol; Group 3: propranolol+pyridostigmine; and Group 4: bisoprolol+ pyridostigmine), using a random number generator at www. random.org. A $2 \times 2$ factorial design was used to compare the efficacy of propranolol and bisoprolol and to assess the benefit of additional pyridostigmine treatment. Patients were instructed to visit the clinic at 1 and 3 months after the initiation of medical treatments. Propranolol was started at a dose of $10 \mathrm{mg}$ twice a day, and a dosage increase was allowed up to $20 \mathrm{mg}$ twice a day after 1 month, according to the clinician's discretion. The starting dose of bisoprolol was $2.5 \mathrm{mg}$ once a day, and a dosage increase was allowed up to $5 \mathrm{mg}$ once a day after 1 month. Pyridostigmine was prescribed at a dose of $30 \mathrm{mg}$ twice a day, which was maintained for 3 months.

\section{Efficacy Assessment}

All participants performed three sets of self-report questionnaires at baseline and at every follow-up visit at 1 and 3 months after the initiation of medical treatment. The OI symptoms were evaluated using the OIQ, which has been widely used to assess the symptoms of POTS patients in previous studies [25-28]. This questionnaire consists of 10 symptoms of OI, which are nausea, tremor in hands, dizziness, palpitations, headache, profuse perspiration, blurred vision, chest discomfort, lightheadedness, and concentration difficulties. Patients specified the presence and frequency of each symptom with a score ranging from 0 to 4 , with 0 for none, 1 for once a month, 2 for 2-4 times per month, 3 for 2-7 times per week, and 4 for more than once a day. The severity of OI symptoms was assessed by summing the scores of the 10 symptoms.

The Beck depression inventory-II (BDI-II) was used to assess the degree of depression in participants. A total score of 0 13 indicates minimal depression, 14-19 mild depression, 20 28 moderate depression, and 29-63 severe depression [29]. 
The health-related QOL in participants was assessed using the 36-item short-form health survey (SF-36) [30]. The questionnaire includes 36 items that produce 8 subscales: physical functioning, role limitation caused by physical problems, bodily pain, general health, vitality, social functioning, role limitations caused by emotional problems, and mental health. These 8 subscale scores are summed into two summary scales, the physical component summary scale (PCS) and the mental component summary scale (MCS). These scales are normed to the population $($ mean $=50$, standard deviation $[\mathrm{SD}]=10)$, and a higher score represents a better QOL, while a lower score indicates a poorer QOL. The changes in clinical scores at each visit were analyzed and the efficacy of medical treatments was investigated according to the treatment regimens.

\section{Outcome Measures}

The primary outcome was the amount of OIQ score reduction after 1 and 3 months of medical treatment compared to the baseline OIQ score. The secondary outcomes included the amount of score reduction in the BDI-II; the amount of score improvement in the PCS; and the amount of score increase in the MCS, which were evaluated after 1 and 3 months of medical treatment. As the orthostatic tachycardia in POTS patients displays diurnal variability $[2,31]$, and the extent of the increase in HR does not correlate with the severity of the symptoms $[8,32]$, changes in the extent of orthostatic tachycardia were not used as an outcome variable.

\section{Statistical Analysis}

All continuous variables are presented as mean \pm SD. Sample size was calculated using the OIQ score distribution obtained from a previous study (estimated mean 19, estimated SD 7) [8]. Sample size was calculated to achieve $80 \%$ power (alpha value 0.05 ) to detect $30 \%$ difference of OIQ score in two different groups. Considering a dropout rate of $20 \%$, we initially designed to perform randomization in 100 patients in the study. However, the dropout rate was higher in some group therefore we enrolled a few more patients.

The difference between the scores of each questionnaire and their changes among treatment groups has been assessed using an analysis of variance (ANOVA) with post hoc analysis performed with the Tukey test. The repeated measures ANOVA with Bonferroni correction was used to compare the data over time (baseline, 1 month, 3 months), in total and within groups. To compare the therapeutic efficacy among different groups, delta changes of the scores from baseline were calculated, and either an ANOVA or an independent $T$ test was performed. Data were analyzed using SPSS version 22.0.0 for Windows, and values of $P<0.05$ were considered significant.

\section{Results}

\section{Patients}

Between April 2014 and August 2015, 153 patients who displayed excessive orthostatic tachycardia ( $\geq 30 \mathrm{bpm}$ increase) were screened for eligibility in the study (Fig. 1). Fifty patients with mild OI symptoms, defined as an OIQ < 10, were excluded. A total of 103 newly diagnosed patients were randomized into 4 different treatment groups and were scheduled to receive 3 months of medical treatment; however, 26 patients discontinued follow-up during the study period. The reason for the discontinuation was due to refusal of further treatments or not showing up at follow-up visits. None of the patients have discontinued follow-up due to adverse events of the medical treatment.

As a result, a total of 77 patients (41 females; mean age $33.0 \pm 12.7)$ who completed the 3 -month follow-up were included in the analysis. In total, the maximal HR increase was $41.9 \pm 10.8$ (Table 1), and the average of the total OIQ, BDI-II, PCS, and MCS scores at baseline was $18.5 \pm 6.7,15.9 \pm 8.2$, $41.5 \pm 6.3$, and $38.5 \pm 10.6$, respectively (Table 2 ).

The number of patients allocated to Groups 1, 2, 3, and 4 was $19,17,18$, and 23, respectively. Baseline demographics, including age, height, weight, supine $\mathrm{HR} /$ systolic BP/diastolic $\mathrm{BP}$, standing HR/systolic BP/diastolic BP (measured immediately after standing), and maximal HR increment after standing were not different among groups (Table 1).

\section{Efficacy}

Regardless of the treatment regimen, the OIQ score significantly decreased after 1 month of medical treatment and further decreased after 3 months of treatment. In total, the mean OIQ decreased from $18.5 \pm 6.7$ to $12.5 \pm 4.5(P<0.01)$ and $7.8 \pm 5.7(P<0.01)$ after 1 and 3 months, respectively. Likewise, in each treatment group, the OI symptoms significantly decreased after 1 month (all $P<0.01$ ) and continued to decrease until 3 months of medical treatment (all $P<0.01$ ). The amount of the decrease in the OIQ score was not different among groups after 1 and 3 months of treatment (all $P>0.05$; Table 2). The improvements of OIQ scores were comparable regardless of the type of $\beta$-blockers (propranolol or bisoprolol) and regardless of adjunctive pyridostigmine treatment (all $P>0.05$; Table 3).

The BDI-II scores decreased in total and in each group after 3 months (all $P<0.01$ ) of medical treatment. The PCS and MCS scores also tended to improve after medical treatment, regardless of the treatment regimen (Table 2). Both propranolol and bisoprolol seemed to be equally helpful for relieving depression and improving QOL in POTS patients (all $P>0.05$ on independent $T$ test, Table 3 ). There seemed to be no incremental benefits of adding pyridostigmine on top of the $\beta$ - 


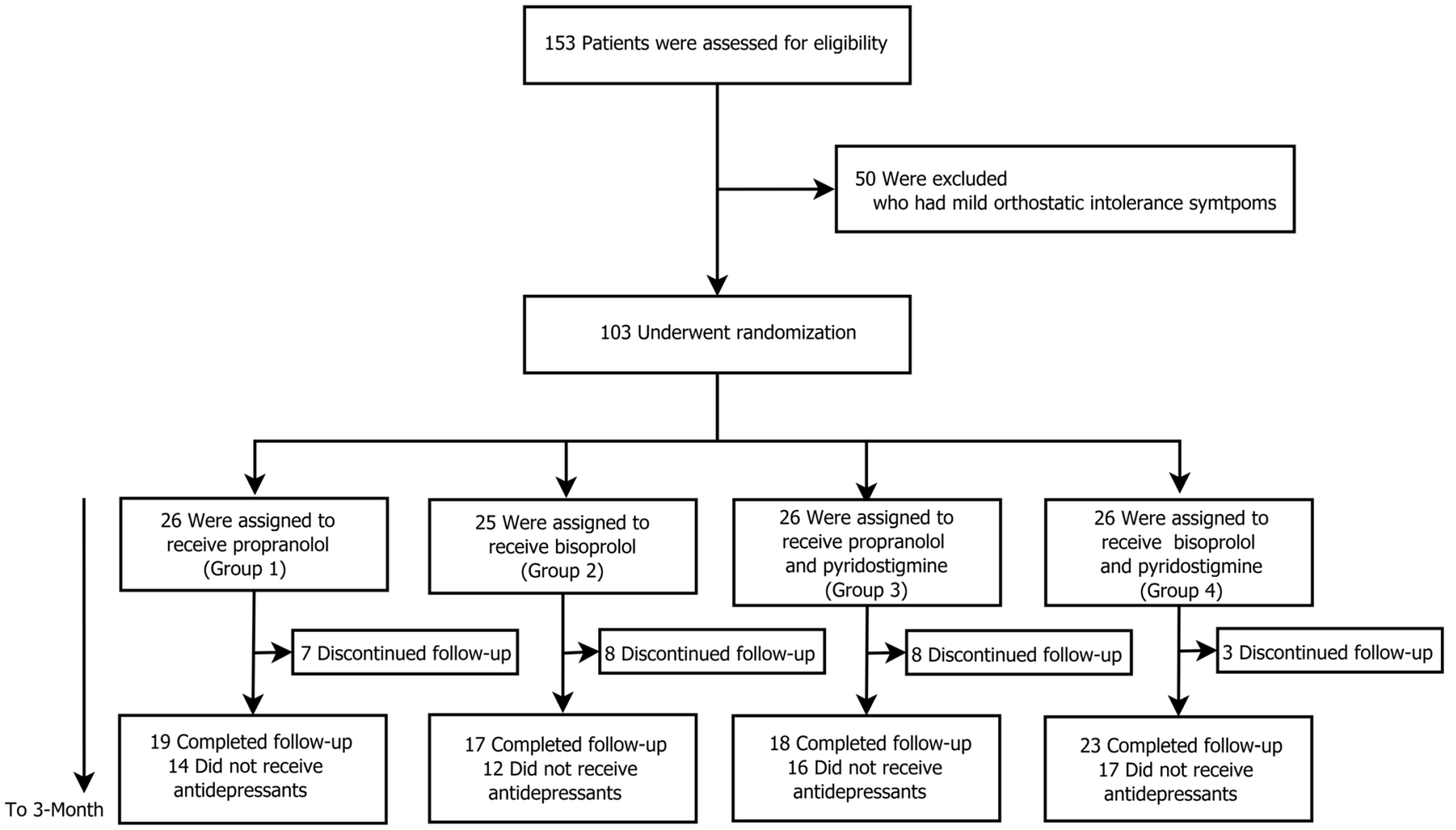

Fig. 1 Screening, randomization, treatment, and follow-up of the patients. Among the 154 patients with excessive orthostatic tachycardia, 50 patients with mild orthostatic intolerance symptoms were excluded.

The remaining 103 patients were randomly assigned to 4 treatment groups. Patients who completed the 3-month medical treatment regimen were included in the analysis

blockers for POTS patients, in terms of managing depression and decreased QOL (all $P>0.05$ on independent $T$ test, Table 3). However, some of the patients had received antidepressants during the study period, which can significantly affect the scores of the BDI-II, PCS, and MCS. Therefore, we assessed the impact of the medical treatment on depression and QOL in the subgroups of patients who did not receive antidepressants during the study period.

\section{Subgroup Analysis}

A total of 59 patients were not treated with antidepressants throughout the study period. The BDI-II scores, along with the OIQ scores, universally decreased after 1 month of medical treatment and further decreased after 3 months of treatment, regardless of the treatment regimen (Fig. 2, Supplementary Table 1). The amount of score changes at 1 and 3 months was not different among groups (Supplementary Table 1). The PCS score significantly increased at 3 months after treatment in every group except ambiguous improvement in Group 2 , and the MCS score remarkably improved in group 3 after 3 months (Fig. 2).

We performed additional subgroup analyses to compare the therapeutic efficacy of propranolol and bisoprolol. Among the patients not treated with antidepressants, patients in Groups 1 and 3 were combined into the propranolol group $(n=30)$, and those in Groups 2 and 4 were united into the bisoprolol group $(n=29)$. The delta changes of each score were compared between the two groups at 1 and 3 months after medical treatment, which displayed no difference between the two treatments (Fig. 3A).

Next, we assessed the benefit of the additional administration of pyridostigmine to $\beta$-blockers. Among the patients not treated with antidepressants, patients in Groups 3 and 4 were merged into the pyridostigmine ( + ) group $(n=33)$, and those in Groups 1 and 2 were combined into the pyridostigmine (-) group $(n=26)$. The delta changes of each score were compared between the two groups at 1 and 3 months after medical treatment, and the additional benefit of pyridostigmine administration was questionable (Fig. 3B).

\section{Discussion}

We evaluated the efficacy of sustained medical treatments in the largest number of POTS patients ever studied. We randomized the patients into 4 different treatment groups to compare the efficacy between two different $\beta$-blockers and to investigate the benefit of additional pyridostigmine treatment. In total, the 3-month medical treatment significantly improved the OI 
Table 1 Baseline characteristics of study patients overall and in each group*

\begin{tabular}{|c|c|c|c|c|c|c|}
\hline & Total & Group 1 (P only) & Group 2 (B only) & Group $3(\mathrm{P}+\mathrm{PS})$ & Group 4 (B + PS) & $P$ value (ANOVA) \\
\hline Number $(N)$ & 77 & 19 & 17 & 18 & 23 & \\
\hline Sex (Male:Female) & $26: 41$ & $6: 13$ & $8: 9$ & $3: 15$ & $9: 14$ & \\
\hline Age & $33.0 \pm 12.7$ & $39.4 \pm 11.6$ & $29.8 \pm 9.9$ & $32.8 \pm 12.8$ & $30.3 \pm 14.0$ & 0.069 \\
\hline Height & $164.6 \pm 9.3$ & $162.0 \pm 8.3$ & $167.9 \pm 8.4$ & $163.0 \pm 9.1$ & $165.7 \pm 10.6$ & 0.211 \\
\hline Weight & $59.9 \pm 13.4$ & $58.0 \pm 9.5$ & $62.2 \pm 14.1$ & $55.3 \pm 11.0$ & $63.4 \pm 16.6$ & 0.211 \\
\hline \multicolumn{7}{|l|}{ Supine } \\
\hline Heart rate ${ }^{*}$ & $65.0 \pm 11.8$ & $64.5 \pm 10.4$ & $64.4 \pm 10.2$ & $67.7 \pm 15.0$ & $63.9 \pm 11.1$ & 0.487 \\
\hline Systolic BP ${ }^{*}$ & $109.1 \pm 11.1$ & $109.1 \pm 12.3$ & $110.0 \pm 10.6$ & $107.3 \pm 9.4$ & $109.9 \pm 11.7$ & 0.697 \\
\hline Diastolic BP* & $66.6 \pm 8.8$ & $68.3 \pm 10.2$ & $66.4 \pm 8.8$ & $66.2 \pm 8.7$ & $65.7 \pm 7.7$ & 0.590 \\
\hline \multicolumn{7}{|l|}{ Standing (immediate) } \\
\hline Heart rate ${ }^{*}$ & $90.7 \pm 15.2$ & $87.1 \pm 13.6$ & $93.5 \pm 14.4$ & $94.2 \pm 16.6$ & $88.8 \pm 15.2$ & 0.112 \\
\hline Systolic BP ${ }^{*}$ & $110.9 \pm 13.0$ & $109.7 \pm 15.4$ & $110.5 \pm 13.0$ & $110.1 \pm 10.3$ & $112.8 \pm 12.8$ & 0.676 \\
\hline Diastolic BP* & $70.0 \pm 11.3$ & $70.4 \pm 10.4$ & $68.9 \pm 10.7$ & $70.2 \pm 10.3$ & $70.1 \pm 13.4$ & 0.937 \\
\hline \multicolumn{7}{|c|}{ Maximal HR incrementII } \\
\hline Overall & $41.9 \pm 10.8$ & $39.3 \pm 13.7$ & $42.6 \pm 9.9$ & $43.8 \pm 10.0$ & $41.9 \pm 9.9$ & 0.641 \\
\hline Afternoon & $25.8 \pm 11.4$ & $22.3 \pm 11.8$ & $28.4 \pm 11.7$ & $29.6 \pm 12.2$ & $23.8 \pm 9.3$ & 0.153 \\
\hline Morning & $40.3 \pm 12.6$ & $38.1 \pm 15.4$ & $40.1 \pm 11.6$ & $41.8 \pm 12.9$ & $41.3 \pm 11.0$ & 0.822 \\
\hline
\end{tabular}

ANOVA was performed among Groups 1 to 4 . Plus-minus values are mean $\pm \mathrm{SD}$

$B P$ blood pressure; $H R$, heart rate; $P$, propranolol; $B$, bisoprolol; $P S$, pyridostigmine; ANOVA, analysis of variance

* The HR and BPs were obtained twice from the 2 orthostatic vital sign tests performed in each patient, and their means were calculated

${ }^{\mathbb{I}}$ The largest value of the maximal heart rate increment was chosen from the 2 orthostatic vital sign tests performed in each patient

symptoms of POTS patients. Moreover, depression and QOL also remarkably improved without the use of antidepressants. Propranolol and bisoprolol demonstrated comparable therapeutic effects for POTS patients. Additional pyridostigmine administration was not beneficial.

The 3-month medical treatments were significantly helpful in improving the OI symptoms of POTS patients. To date, non-pharmacological treatments including water/sodium ingestion and exercise are prescribed to POTS patients, and several medications have been accepted for the pharmacological treatments of POTS. Randomized trials have elucidated that low-dose propranolol [14], midodrine [22], and pyridostigmine [17] are beneficial for the improvement of orthostatic tachycardia and OI symptoms. However, most of these drug trials are designed to evaluate the acute response (2 to $4 \mathrm{~h}$ after administration) of the study drug $[13,14,17,22]$. Only a limited number of studies have evaluated the sustained effect of daily medical treatment $[18,19,33]$ or exercise training [34] in POTS patients, but these studies were mainly focused on the improvement of haemodynamic parameters [19, $23,35]$. Recent studies have revealed that orthostatic tachycardia displays diurnal variability $[2,31]$, and the extent of orthostatic tachycardia does not correlate with the severity of the symptoms [8, 32]; thus, we insist that it should not be used as an outcome variable for evaluating the long-term effect of a medical treatment. We demonstrated that the OI symptoms of
POTS patients significantly decreased after 1 month of medical treatments that consisted of a $\beta$-blocker with/without pyridostigmine. The symptoms continued to decrease in response to 3 months of medical treatment. We suggest that pharmacological treatment of POTS should be maintained for at least 3 months.

Medical treatments without antidepressant agents in POTS patients were helpful in improving the symptoms of depression and diminished QOL. Recently, we have reported that depression and diminished QOL in POTS patients are directly associated with their OI symptoms [8]. However, it has remained unknown if the depressive symptoms of POTS patients need to be treated with additional antidepressant agents or if the depression would be improved after the medical treatment of POTS alone. Among the 59 patients who did not receive antidepressant agents during the study period, the mean BDI-II scores of the patients in each treatment group were all above 13, which suggested the presence of mild depression in the patients. The BDI-II score universally decreased to below 10 after 3 months in each group, which represented minimal depression. The baseline PCS and MCS scores in each treatment group were all below 43 , which indicated that the mean QOL score of the patients belonged to the lowest quartile of the general population. After 3 months of medical treatment, the PCS score significantly improved in every group and the MCS score meaningfully increased in 


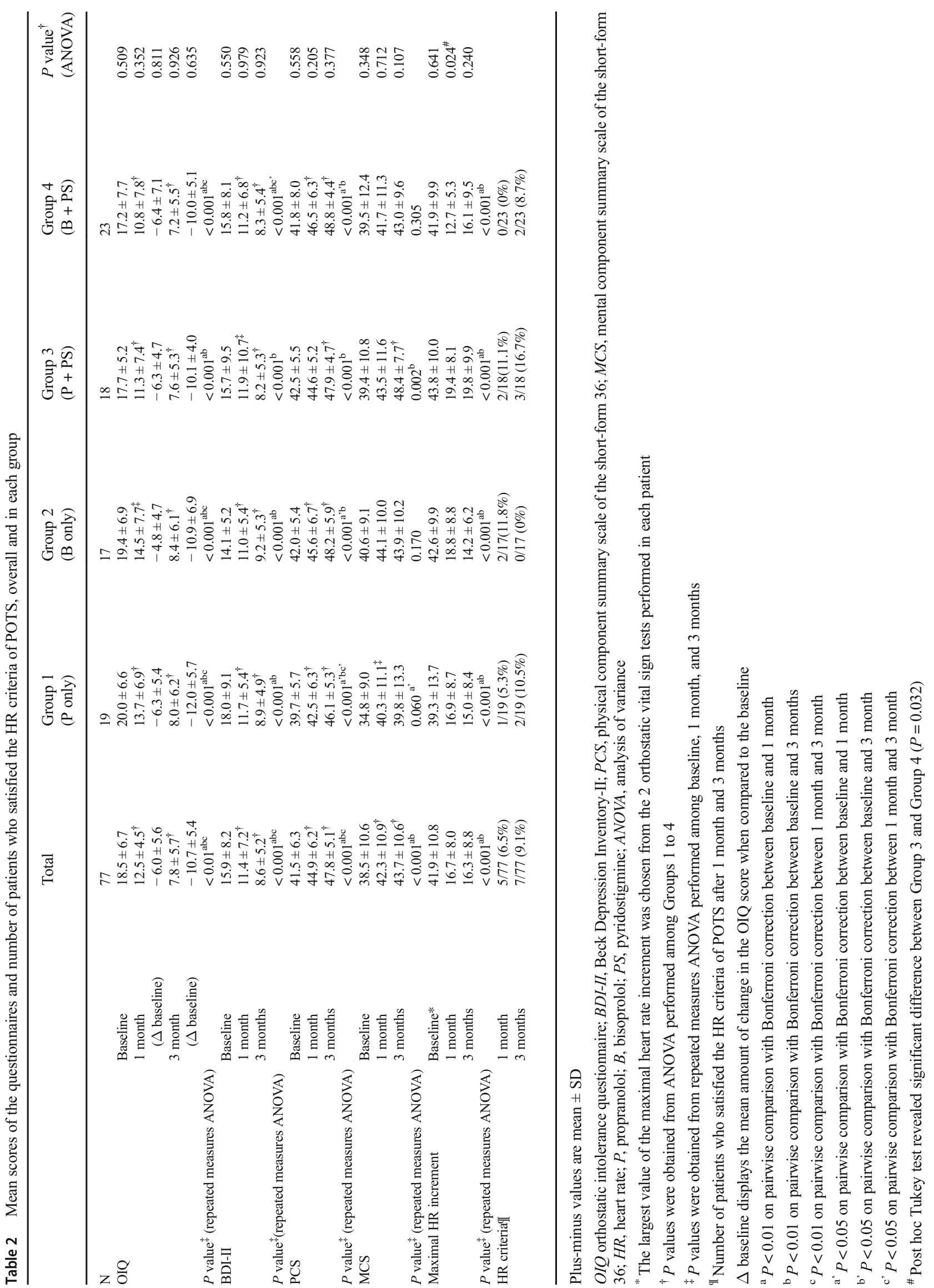




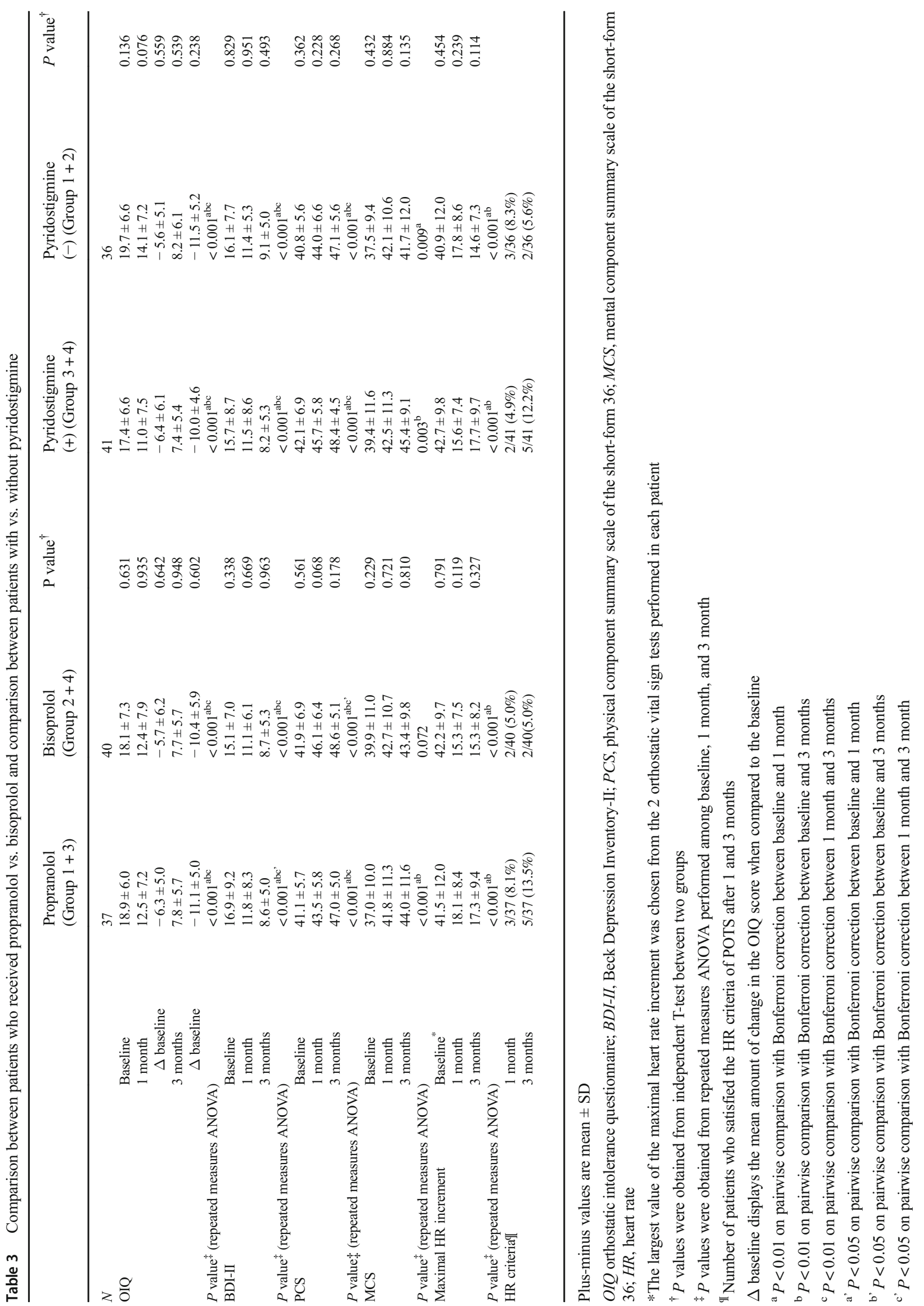


Group 1 (P only, n=14)

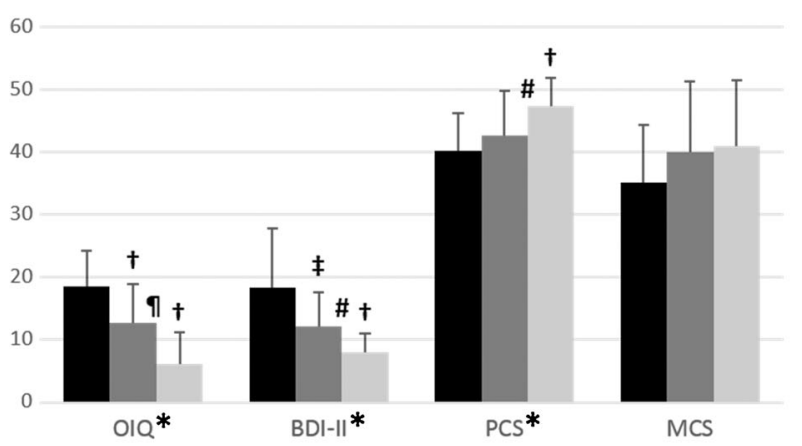

Group 3 (P+PS, n=16)

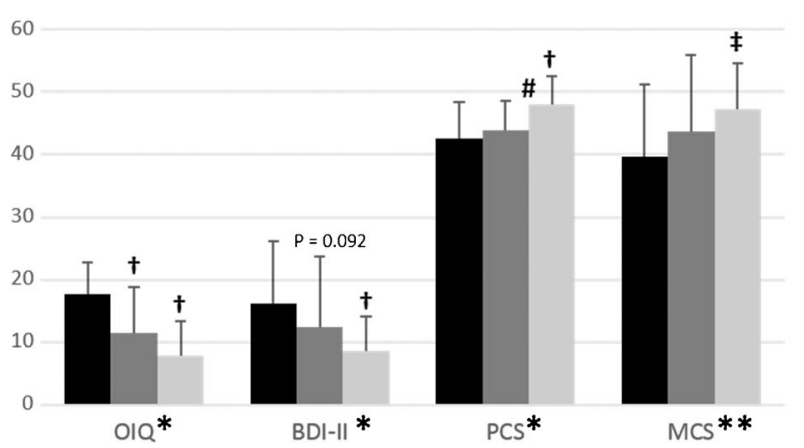

Fig. 2 Subgroup analysis of the patients who did not receive antidepressants during the follow-up period. Regardless of the treatment group, all the patients demonstrated noticeable improvement of their orthostatic intolerance symptoms (measured by the OIQ) and depression (measured by the BDI-II) after 1 month, with further improvement after 3 months of medical treatment. Patients in every treatment group experienced improvement of the physical component of quality of life (measured by PCS) after 3 months, except ambiguous improvement in Group 2. Patients in Group 3 (who received propranolol and pyridostigmine) showed an improvement of the mental component of quality of life (measured by the MCS) after 3 months. P and B indicate propranolol and bisoprolol, respectively; PS, pyridostigmine; OIQ,

Group 3. The current study implies that depression and diminished QOL can be improved by the sustained medical treatment of POTS without antidepressant agents.

Propranolol and bisoprolol were equally effective for improving the OI symptoms, depressive symptoms, and diminished QOL in POTS patients. Propranolol is a nonselective $\beta$-blocker that may induce systemic effects, whereas bisoprolol is a $\beta 1$-selective $\beta$-blocker that may mainly affect the heart $[4,15]$. It has yet to be elucidated whether POTS is merely a disease of the heart or a systemic disease caused by autonomic dysregulation. The changes in clinical scores in the propranolol and bisoprolol groups were not significantly different after 1 and 3 months. However, the amount of improvement in the scores in each scale after 3 months was uniformly larger in the propranolol group despite the lack of statistical significance. The efficacy of propranolol and bisoprolol in POTS patients should be evaluated for a longer period of time in future studies.
Group 2 (B only, $\mathrm{n}=12$ )

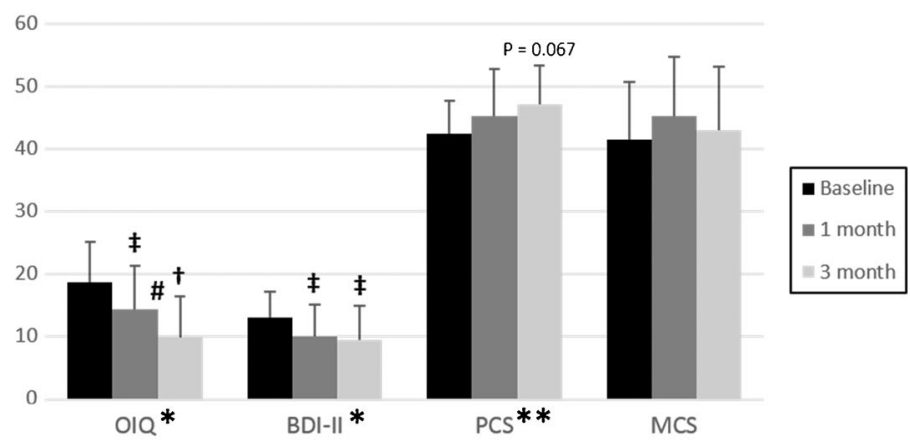

Group 4 (B+PS, n=17)

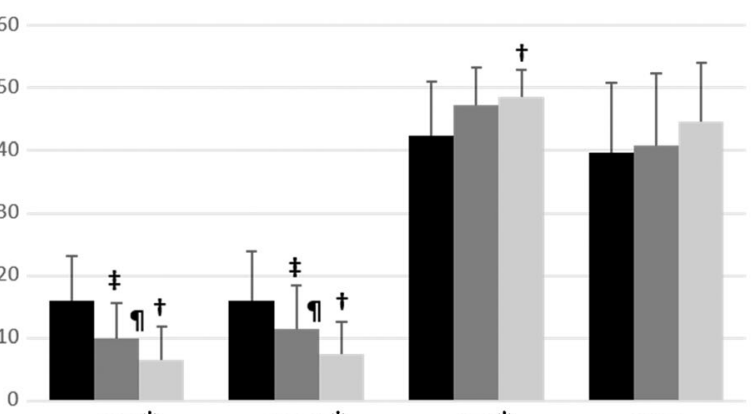

OIQ *

$\mathrm{BDI}-\mathrm{II} *$

PCS*

MCS

orthostatic intolerance questionnaire; BDI-II, Beck Depression Inventory-II; PCS, physical component summary scale of the ShortForm 36; MCS, mental component summary scale of the Short-Form 36. ${ }^{*} P$ values $<0.01$ on repeated measures ANOVA performed among baseline, 1 month, and 3 months. $* * P$ values $<0.05$ on repeated measures ANOVA performed among baseline, 1 month, and 3 month. ${ }^{\dagger} P$ values < 0.01 on pairwise comparison with Bonferroni correction compared to the baseline. ${ }^{\star} P$ values $<0.05$ on pairwise comparison with Bonferroni correction compared to the baseline. ${ }^{\text {II }} P$ values $<0.01$ on pairwise comparison with Bonferroni correction between 1 and 3 months. ${ }^{\#} P$ values $<0.05$ on pairwise comparison with Bonferroni correction between 1 and 3 months

The additional administration of pyridostigmine with $\beta$ blockers in POTS patients was not superior to the treatment with a $\beta$-blocker alone. $\beta$-blockers and pyridostigmine are the most commonly prescribed medications in POTS patients $[4$, $12,36]$. $\beta$-blockers are helpful in reducing the HR in POTS patients [12], but excessive $\beta$-blockade could be counterproductive and less well-tolerated. Low-dose propranolol (10 $20 \mathrm{mg}$ orally) was more beneficial than high-dose propranolol $(80 \mathrm{mg}$ ) for lowering the standing HR and improving the symptoms in POTS patients [14]. Pyridostigmine is an acetylcholinesterase inhibitor that can increase synaptic acetylcholine levels at both the autonomic ganglia and peripheral muscarinic parasympathetic receptors [12]. As $\beta$-blockers and pyridostigmine have different mechanisms of action, we hypothesized that combining the two drugs would be more effective in POTS patients. However, there were no significant differences in score changes in each questionnaire between patients with or without the additional administration of pyridostigmine. 
Fig. 3 Subgroup analysis of the patients combined according to the type of $\beta$-blockers or the presence of pyridostigmine.

Among the patients who did not receive antidepressants throughout the follow-up period, patients were combined according to the choice of $\beta$ blocker or the presence of pyridostigmine. The delta changes of each clinical scale after 1- and 3-month medical treatment regimens are displayed (mean $\pm \mathrm{SD}$ ). The propranolol group designates the combined cohort (Groups 1 and 3) of patients who received propranolol, and the bisoprolol group designates the merged cohort (Groups 2 and 4) of patients who received bisoprolol as the choice of $\beta$-blocker (A). There was no significant difference in efficacy between the two $\beta$-blockers. Pyridostigmine $(+)$ group refers to the combined cohort (Groups 3 and 4) of patients who received pyridostigmine with a $\beta$-blocker, while the pyridostigmine (-) group refers to the combined cohort (Groups 1 and 2) of patients who were treated with $\beta$ blockers only (B). There was no significant difference in efficacy according to the presence of pyridostigmine (all $P>0.05$ )

\section{A}

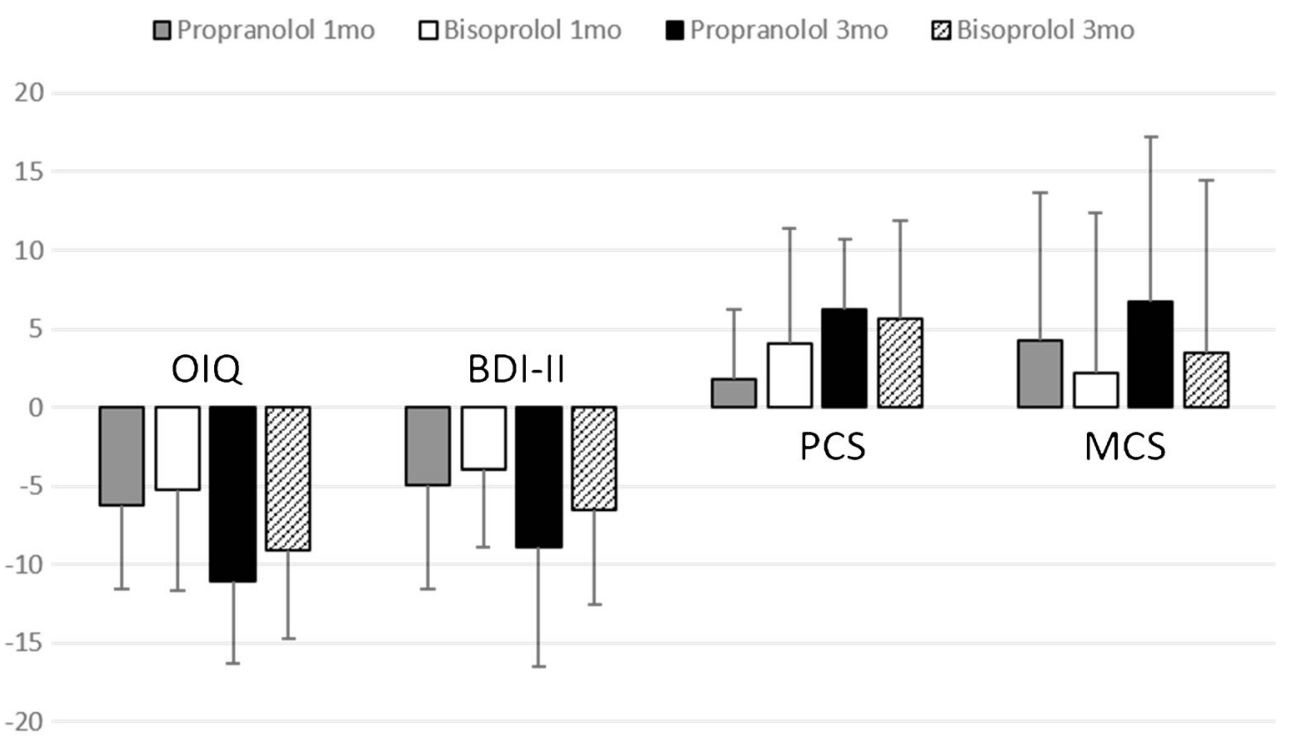

B Pyridostigmine (+) vs. Pyridostigmine (-)

口Pyridostigmine(+) 1mo पPyridostigmine(-) 1mo Pyridostigmine(+) 3mo שPyridostigmine(-) 3mo

20

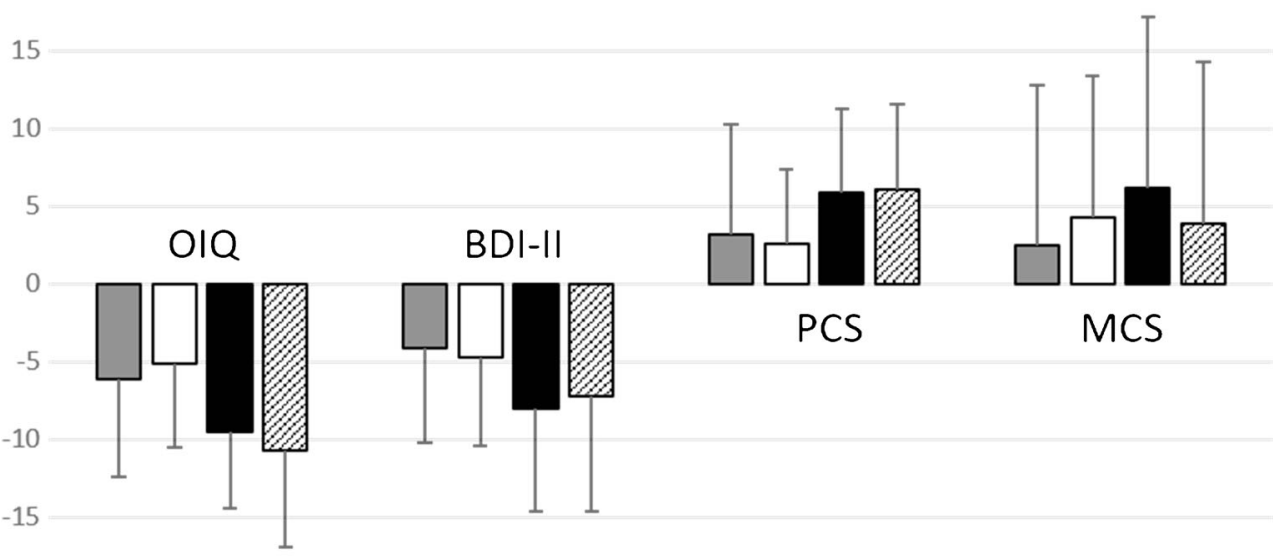

$-20$
Recently, an autoimmune origin of POTS has been suggested, according to the reports that revealed that various autoantibodies were present in POTS patients. Li et al. demonstrated that the anti- $\beta 1$ adrenergic receptor (AR) antibody, anti- $\beta 2$ AR antibody, and anti- $\alpha 1 \mathrm{AR}$ antibody were all present in the serum of POTS patients and that these sera acted as a partial $\alpha 1$ $\mathrm{AR}$ antagonist and as $\beta 1 \mathrm{AR}$ and $\beta 2 \mathrm{AR}$ agonists [5]. Other studies have reported the presence of autoantibodies to acetylcholine receptors (AchRs) [37] and cardiac lipid raft-associated proteins [38]. POTS patients also had a higher prevalence of comorbid autoimmune disorders and autoimmune markers, such as antinuclear antibodies and antiphospholipid antibodies [39]. However, it still remains unknown if the POTS patients would show different responses to various medications according to their autoantibody status. Theoretically, patients with anti- $\beta 1 / 2$ AR antibodies would benefit from $\beta$-blockers and patients with AchR antibodies would respond well to pyridostigmine. The efficacy of these kinds of tailored treatments, according to autoantibody status, needs to be investigated in further studies.

Our study has several strengths, including the prospective design, randomized trial, and large number of participants. Also, we have assessed comprehensive list of symptoms on every visit, including depression and QOL, which enables to assess the clinical improvement in daily living of patients. However, the study also has limitation that there was no 
placebo control group. Although all the medications are used off-label in POTS patients, growing number of evidences suggest that medical treatments are useful in POTS patients. So, we thought including placebo control group for 3 months was unethical. On the other hand, emerging literatures have suggested the benign long-term outcomes of POTS $[40,41]$ and the possibility of placebo effects in clinical trials due to psychological benefits obtained by perceived therapy [42]. It can be possible that POTS patients got better as they have been diagnosed and counseled about their symptoms by a knowledgeable physician or just following the natural course of the disease. Thus, succeeding clinical trials with a placebo group will be required to verify the current findings.

In summary, we investigated the efficacy of sustained medical treatments in the largest number of POTS patients ever studied. We have shown that a 3-month medical treatment regimen was beneficial for patients with POTS, not only for reducing OI symptoms but also for improving depression and diminished QOL, even without prescriptions for antidepressants. The therapeutic efficacy of propranolol and bisoprolol was comparable, and the advantage of the additional administration of pyridostigmine with a $\beta$-blocker was questionable.

Acknowledgments This study was supported by the National Research Foundation of Korea (NRF) funded by the Ministry of Science, ICT \& Future Planning (NRF-2016R1C1B2016275). K.C. was supported by Seoul National University Hospital (0620164080, 0620143970).

Required Author Forms Disclosure forms provided by the authors are available with the online version of this article.

Author Contributions J.M., S.-T.L., K.-H.J., K.-I.P., K.-Y.J., M.K., S.K.L., and K.C. contributed to study concept and design. All authors contributed to data acquisition and analysis. J.M., S.K.L., and K.C. drafted the manuscript.

\section{Compliance with Ethical Standards}

Conflict of Interest The authors declare that they have no competing interests.

\section{References}

1. Benarroch EE, editor. Postural tachycardia syndrome: a heterogeneous and multifactorial disorder. Mayo Clinic Proceedings; 2012: Elsevier.

2. Moon J, Lee HS, Byun J-I, Sunwoo J-S, Shin J-W, Lim J-A et al. The complexity of diagnosing postural orthostatic tachycardia syndrome: influence of the diurnal variability. Journal of the American Society of Hypertension. 2016;10(3):263-70.

3. Sheldon RS, Grubb 2nd B, Olshansky B, Shen W-K, Calkins H, Brignole M et al. 2015 Heart Rhythm Society Expert Consensus Statement on the Diagnosis and Treatment of Postural Tachycardia Syndrome, Inappropriate Sinus Tachycardia, and Vasovagal Syncope. Heart rhythm: the official journal of the Heart Rhythm Society. 2015;12(6):e41-63.

4. Mathias CJ, Low DA, Iodice V, Owens AP, Kirbis M, Grahame R. Postural tachycardia syndrome-current experience and concepts.
Nature Reviews Neurology. 2012;8(1):22-34. doi:https://doi.org/ 10.1038/nrneurol.2011.187.

5. Li H, Yu X, Liles C, Khan M, Vanderlinde-Wood M, Galloway A et al. Autoimmune basis for postural tachycardia syndrome. Journal of the American Heart Association. 2014;3(1):e000755.

6. Bagai K, Song Y, Ling J, Malow B, Black B, Biaggioni I et al. Sleep disturbances and diminished quality of life in postural tachycardia syndrome. Journal of clinical sleep medicine: JCSM: official publication of the American Academy of Sleep Medicine. 2011;7(2):204.

7. Reynolds G, Lewis D, Richardson A, Lidbury B. Comorbidity of postural orthostatic tachycardia syndrome (POTS) and chronic fatigue syndrome (CFS) in an Australian cohort. Journal of internal medicine. 2014;275(4):409-17.

8. Moon J, Kim D-Y, Byun J-I, Sunwoo J-S, Lim J-A, Kim T-J et al. Orthostatic intolerance symptoms are associated with depression and diminished quality of life in patients with postural tachycardia syndrome. Health and quality of life outcomes. 2016;14(1):144.

9. Ruzieh M, Baugh A, Dasa O, Parker RL, Perrault JT, Renno A et al. Effects of intermittent intravenous saline infusions in patients with medication - refractory postural tachycardia syndrome. Journal of Interventional Cardiac Electrophysiology. 2017:1-6.

10. Kanjwal K, Saeed B, Karabin B, Kanjwal Y, Grubb BP. Use of methylphenidate in the treatment of patients suffering from refractory postural tachycardia syndrome. American journal of therapeutics. 2012;19(1):2-6.

11. Khan M, Ouyang J, Perkins K, Somauroo J, Joseph F. Treatment of refractory postural tachycardia syndrome with subcutaneous octreotide delivered using an insulin pump. Case reports in medicine. 2015;2015.

12. Raj SR. Postural Tachycardia Syndrome (POTS). Circulation. 2013;127(23):2336-42. doi:https://doi.org/10.1161/circulationaha. 112.144501

13. Arnold AC, Okamoto LE, Diedrich A, Paranjape SY, Raj SR, Biaggioni I et al. Low-dose propranolol and exercise capacity in postural tachycardia syndrome A randomized study. Neurology. 2013;80(21):1927-33.

14. Raj SR, Black BK, Biaggioni I, Paranjape SY, Ramirez M, Dupont WD et al. Propranolol Decreases Tachycardia and Improves Symptoms in the Postural Tachycardia Syndrome Less Is More. Circulation. 2009;120(9):725-34.

15. Freitas J, Santos R, Azevedo E, Costa O, Carvalho M. Clinical improvement in patients with orthostatic intolerance after treatment with bisoprolol and fludrocortisone. Clinical Autonomic Research. 2000;10(5):293-9.

16. Zhao J, Du S, Yang J, Lin J, Tang C, Du J et al. Usefulness of plasma copeptin as a biomarker to predict the therapeutic effectiveness of metoprolol for postural tachycardia syndrome in children. The American journal of cardiology. 2014;114(4):601-5.

17. Raj SR, Black BK, Biaggioni I, Harris PA, Robertson D. Acetylcholinesterase inhibition improves tachycardia in postural tachycardia syndrome. Circulation. 2005;111(21):2734-40.

18. Chen L, Wang L, Sun J, Qin J, Tang C, Jin H et al. Midodrine hydrochloride is effective in the treatment of children with postural orthostatic tachycardia syndrome. Circulation Journal. 2011;75(4):927-31.

19. Ross AJ, Ocon AJ, Medow MS, Stewart JM. A double-blind placebo-controlled cross-over study of the vascular effects of midodrine in neuropathic compared with hyperadrenergic postural tachycardia syndrome. Clinical Science. 2014;126(4):289-96.

20. Yang JY, Zhao J, Du SX, Liu D, Fu CH, Li XY et al. Postural Orthostatic Tachycardia Syndrome with Increased Erythrocytic Hydrogen Sulfide and Response to Midodrine Hydrochloride. Journal of Pediatrics. 2013;163(4):1169-+. doi:https://doi.org/10. 1016/j.jpeds.2013.04.039.

21. Lai CC, Fischer PR, Brands CK, Fisher JL, PORTER CBJ, Driscoll SW et al. Outcomes in Adolescents with Postural Orthostatic 
Tachycardia Syndrome Treated with Midodrine and $\beta$-Blockers. Pacing and Clinical Electrophysiology. 2009;32(2):234-8.

22. Hoeldtke RD, Bryner KD, Hoeldtke ME, Hobbs G. Treatment of postural tachycardia syndrome: a comparison of octreotide and midodrine. Clinical Autonomic Research. 2006;16(6):390-5.

23. Green EA, Black BK, Biaggioni I, Paranjape SY, Bagai K, Shibao $\mathrm{C}$ et al. Melatonin reduces tachycardia in postural tachycardia syndrome: a randomized, crossover trial. Cardiovascular therapeutics. 2014;32(3):105-12.

24. Kanjwal K, Karabin B, Sheikh M, Elmer L, Kanjwal Y, Saeed B et al. Pyridostigmine in the Treatment of Postural Orthostatic Tachycardia: A Single-Center Experience. Pacing and Clinical Electrophysiology. 2011;34(6):750-5.

25. Winker R, Barth A, Dorner W, Mayr O, Pilger A, Ivancsits S et al. Diagnostic management of orthostatic intolerance in the workplace. International archives of occupational and environmental health. 2003;76(2):143-50.

26. Winker R, Barth A, Bidmon D, Ponocny I, Weber M, Mayr O et al. Endurance exercise training in orthostatic intolerance : A randomized, controlled trial. Hypertension. 2005;45(3):391-8. doi:https:// doi.org/10.1161/01.HYP.0000156540.25707.af.

27. Liao Y, Yang JY, Zhang FW, Chen S, Liu XQ, Zhang QY et al. Flow-Mediated Vasodilation as a Predictor of Therapeutic Response to Midodrine Hydrochloride in Children With Postural Orthostatic Tachycardia Syndrome. Am J Cardiol. 2013;112(6): 816-20. doi:https://doi.org/10.1016/j.amjcard.2013.05.008.

28. Zhang F, Li X, Ochs T, Chen L, Liao Y, Tang C et al. Midregional pro-adrenomedullin as a predictor for therapeutic response to midodrine hydrochloride in children with postural orthostatic tachycardia syndrome. Journal of the American College of Cardiology. 2012;60(4):315-20.

29. Beck AT, Steer RA, Ball R, Ranieri WF. Comparison of Beck Depression Inventories-IA and-II in psychiatric outpatients. Journal of personality assessment. 1996;67(3):588-97.

30. Jenkinson C, Coulter A, Wright L. Short form 36 (SF36) health survey questionnaire: normative data for adults of working age. Bmj. 1993;306(6890):1437-40.

31. Brewster JA, Garland EM, Biaggioni I, Black BK, Ling JF, Shibao CA et al. Diurnal variability in orthostatic tachycardia: implications for the postural tachycardia syndrome. Clinical science (London, England : 1979). 2012;122(1):2531. doi:https://doi.org/10.1042/cs20110077.
32. Kimpinski K, Figueroa JJ, Singer W, Sletten DM, Iodice V, Sandroni P et al., editors. A Prospective, 1-Year Follow-up Study of Postural Tachycardia Syndrome. Mayo Clinic Proceedings; 2012: Elsevier.

33. Yang J, Zhao J, Du S, Liu D, Fu C, Li X et al. Postural orthostatic tachycardia syndrome with increased erythrocytic hydrogen sulfide and response to midodrine hydrochloride. The Journal of pediatrics. 2013;163(4):1169-73. e2.

34. Fu Q, VanGundy TB, Shibata S, Auchus RJ, Williams GH, Levine BD. Exercise training versus propranolol in the treatment of the postural orthostatic tachycardia syndrome. Hypertension. 2011;58(2):167-75.

35. Stewart JM, Munoz J, Weldon A. Clinical and physiological effects of an acute $\alpha-1$ adrenergic agonist and a $\beta-1$ adrenergic antagonist in chronic orthostatic intolerance. Circulation. 2002;106(23):2946-54.

36. Thieben MJ, Sandroni P, Sletten DM, Benrud-Larson LM, Fealey $\mathrm{RD}$, Vernino $\mathrm{S}$ et al., editors. Postural orthostatic tachycardia syndrome: the Mayo clinic experience. Mayo Clinic Proceedings; 2007: Elsevier.

37. Li J, Zhang Q, Liao Y, Zhang C, Hao H, Du J. The value of acetylcholine receptor antibody in children with postural tachycardia syndrome. Pediatric cardiology. 2015;36(1):165-70.

38. Wang X-L, Ling T-Y, Charlesworth MC, Figueroa JJ, Low P, Shen $\mathrm{W}-\mathrm{K}$ et al. Autoimmunoreactive IgGs against cardiac lipid raftassociated proteins in patients with postural orthostatic tachycardia syndrome. Translational Research. 2013;162(1):34-44.

39. Blitshteyn S. Autoimmune markers and autoimmune disorders in patients with postural tachycardia syndrome (POTS). Lupus. 2015;24(13):1364-9.

40. Sousa A, Lebreiro A, Freitas J, Maciel MJ. Long-term follow-up of patients with postural tachycardia syndrome. Clinical Autonomic Research. 2012;22(3):151-3.

41. Bhatia R, Kizilbash SJ, Ahrens SP, Killian JM, Kimmes SA, Knoebel EE et al. Outcomes of adolescent-onset postural orthostatic tachycardia syndrome. The Journal of pediatrics. 2016;173:149-53.

42. Nwazue VC, Arnold AC, Raj V, Black BK, Biaggioni I, Paranjape SY et al. Understanding the placebo effect in clinical trials for postural tachycardia syndrome. Clinical and experimental pharmacology \& physiology. 2014;41(5):32530. doi:https://doi.org/10.1111/1440-1681.12221. 